\title{
Segurança alimentar e nutricional e variáveis associadas em famílias de usuários de terapia nutricional enteral domiciliar
}

\author{
Food and nutritional security and associated variables in families of home enteral nutrition therapy
}

Seguridad alimentaria y nutricional y variables asociadas en familias de terapia de nutrición

enteral en el hogar

Recebido: 05/11/2021 | Revisado: 12/11/2021 | Aceito: 14/11/2021 | Publicado: 22/11/2021

\author{
Caryna Eurich Mazur \\ ORCID: https://orcid.org/0000-0002-1278-5963 \\ Universidade Estadual do Centro-Oeste, Brasil \\ E-mail: carynanutricionista@gmail.com \\ Marcia Maria Arenhart Soares \\ ORCID: https://orcid.org/0000-0001-6081-9550 \\ Universidade Federal do Paraná, Brasil \\ E-mail: marcia.arenharts@gmail.com \\ Maria Eliana Madalozzo Schieferdecker \\ ORCID: https://orcid.org/0000-0002-9400-3717 \\ Universidade Federal do Paraná, Brasil \\ E-mail: meliana.ufpr@gmail.com
}

\begin{abstract}
Resumo
A terapia nutricional enteral domiciliar (TNED) é uma modalidade de atenção nutricional realizada no domicílio. A TNED converge para os princípios de segurança alimentar e nutricional (SAN); pelo ato de não comer pela boca o paciente, por vezes, é acometido pela piora do quadro de SAN. Assim, esse estudo tem por objetivo avaliar a SAN e associar com demais variáveis socioeconômicas, clínicas e nutricionais em pacientes em TNED. Trata-se de uma pesquisa transversal. Foram avaliados dados socioeconômicos, clínicos e nutricionais, além da verificação da SAN por questionário validado. Participaram da pesquisa 45 indivíduos, com média de idade de 74,56士11,86 anos; 46,7\% (n=21) dos domicílios apresentavam Insegurança Alimentar e Nutricional (IAN) e todos foram categorizados com IAN leve. Somente a idade e renda per capita foram significativamente diferentes quando comparados os perfis dos domicílios com SAN e IAN $(\mathrm{p}<0,05)$. A SAN preexistente nos domicílios dos doentes não foi considerada variável preditora independente para a categoria de fórmula administrada, mesmo quando ajustado para outras variáveis $(\mathrm{p}>0,05)$. Assim, nem a SAN tampouco a IAN familiares, associaram-se com as demais variáveis apresentadas pelos pacientes em TNED. Palavras-chave: Continuidade da assistência ao paciente; Programas sociais; Serviços de assistência domiciliar.
\end{abstract}

\begin{abstract}
Home enteral nutritional therapy (HENT) is a modality of nutritional care provided at home. HENT converges on the principles of food and nutrition security (FNS); due to the act of not eating by mouth, the patient is sometimes affected by the worsening of the FNS condition. Thus, this study aims to evaluate FNS and associate it with other variables in HENT patients. This is a cross-sectional survey. Socioeconomic, clinical and nutritional data were evaluated, in addition to the FNS verification by a validated questionnaire. 45 individuals participated in the research, with a mean age of $74.56 \pm 11.86$ years; $46.7 \%(\mathrm{n}=21)$ of the households had Food and Nutritional Insecurity (FNI) and all were categorized with mild FNI. Only age and per capita income were significantly different when comparing the profiles of households with FNS and FNI $(\mathrm{p}<0.05)$. The preexisting FNS in the patients' homes was not considered an independent predictor variable for the category of formula administered, even when adjusted for other variables ( $>0.05)$. Thus, neither FNS nor family FNI were associated with the other variables presented by patients in HENT.
\end{abstract}

Keywords: Continuity of patient care; Social programs; Home assistance services.

\section{Resumen}

La terapia nutricional enteral domiciliaria (TNED) es una modalidad de atención nutricional que se brinda en el hogar. TNED converge en los principios de seguridad alimentaria y nutricional (SAN); debido al acto de no comer por vía oral, el paciente a veces se ve afectado por el empeoramiento de la condición de SAN. Así, este estudio tiene como objetivo evaluar la SAN y asociarla con otras variables en pacientes con TNED. Esta es una encuesta transversal. Se evaluaron datos socioeconómicos, clínicos y nutricionales, además de la verificación de la SAN mediante un cuestionario validado. En la investigación participaron 45 individuos, con una edad media de 74,56 $\pm 11,86$ años; El $46,7 \%(n=21)$ de los hogares presentaba Inseguridad Alimentaria y Nutricional (IAN) y todos fueron categorizados con IAN leve. Solo la edad y el ingreso per cápita fueron significativamente diferentes al comparar los perfiles de 
hogares con SAN e IAN ( $\mathrm{p}<0.05$ ). La SAN preexistente en los domicilios de los pacientes no se consideró variable predictora independiente para la categoría de fórmula administrada, aun cuando se ajustó por otras variables (p>0,05). Así, ni la SAN ni la IAN familiar se asociaron al resto de variables presentadas por los pacientes en TNED.

Palabras clave: Continuidad de la atención al paciente; Programas sociales; Servicios de asistencia domiciliaria.

\section{Introdução}

A Segurança Alimentar e Nutricional (SAN) é baseada na produção e formação de estoques de alimentos além da influência do sistema alimentar nas condições de vida dos indivíduos, associada à realização do direito ao acesso aos alimentos de qualidade, em quantidade suficiente, sem comprometer outras necessidades essenciais. Assim, se faz necessária a implementação de políticas e programas de vários setores com capacidades para promover, na dimensão individual e coletiva, o acesso à alimentação para garantia do Direito Humano à Alimentação e Nutrição Adequadas (Vasconcellos \& Moura, 2018; Mizzuno et al., 2021). A SAN em domicílio deve ser focada sobretudo no indivíduo que está com alimentação especial. A SAN conceitualmente também se refere ao bem estar físico, social, ambiental, além da qualidade de vida (Kepple; Segall-Corrêa, 2011; Mazur et al., 2014).

A Terapia Nutricional Enteral Domiciliar (TNED) é uma modalidade de cuidado em franca expansão, especialmente quando pensado que o doente pode ter maior contato com o ambiente familiar. Comumente, no processo de desospitalização os indivíduos em TNED e seus cuidadores recebem a prescrição de fórmulas comerciais e uma receita/fórmula com alimentos liquidificados como escolha, na impossibilidade de adquirir a fórmula industrializada. É desconhecida, até então, a associação entre a SAN plena e a utilização de fórmulas industrializadas ou com alimentos, possivelmente ao proporcionar confiança ao doente e seu cuidador/família. Sabe-se que os alimentos têm teores maiores de compostos bioativos que as fórmulas industrializadas, além das fibras estarem mais presentes. Também, é preciso ponderar o contexto social que o alimento exerce o qual é mais forte (Poulain, 2004; ADA, 2013; Mazur et al., 2014).

$\mathrm{O}$ acidente vascular cerebral (AVC) é uma das doenças com maior mortalidade no mundo, ademais acarreta em morbidades relacionadas à dependência de cuidados em geral. Indivíduos que sofreram AVC estão sujeitos a alterações neurológicas e cognitivas que afetam tecidos musculares, maiormente os músculos pertinentes à deglutição, o que leva à impossibilidade de alimentarem-se pela boca. Nesse sentido, a opção é a utilização da terapia nutricional enteral (TNE), método amplamente aconselhado para alimentação de indivíduos que não podem se alimentar por via oral (Ojo \& Brooke, 2016; Mazur et al., 2021).

Tendo em vista os fatores de risco para desnutrição primária pela má alimentação, administração inadequada da TNE, limitação socioeconômica e para a desnutrição secundária provocada pelo AVC, se faz necessário verificar a SAN no contexto familiar e individual no domicílio. De tal modo, o objetivo do presente estudo foi relacionar a SAN e variáveis clínicas, nutricionais e socioeconômicas em pacientes com diagnóstico prévio de AVC em TNED.

\section{Metodologia}

Trata-se de um estudo transversal. Foram inclusos no estudo indivíduos adultos e idosos avaliados e acompanhados pelos nutricionistas responsáveis pelas Unidades Básicas de Saúde (UBS) da Secretaria Municipal de Saúde (SMS) de Curitiba, Paraná, durante maio a dezembro de 2013. Este estudo foi aprovado pelo Comitê de Ética da Universidade Federal do Paraná (Parecer 197.447 / CAAE: 11357712.5.3001.0101). Todos os indivíduos que sofreram AVC e participavam do Programa de Atenção Nutricional a Pessoas com Necessidades Alimentares Especiais (PAN) foram convidados a participar do estudo; ao final a amostra foi de 45 indivíduos.

Foram coletados dados socioeconômicos (escolaridade do indivíduo; renda mensal familiar com o número de salários mínimos (valor no ano de 2013 de R\$ 678,00); participação em programas governamentais; número de pessoas que vivem no 
domicílio e quantas trabalham; estado civil do indivíduo; e se o cuidador possui vínculo empregatício).

Para avaliar a SAN do domicílio foi aplicada a Escala Brasileira de Insegurança Alimentar e Nutricional (EBIA), versão adaptada à realidade brasileira (Bickel et al., 2000; Perez-Escamilla et al., 2004). A EBIA foi respondida pelo cuidador ou chefe da família. A EBIA admite a classificação dos domicílios em quatro categorias: segurança alimentar, IAN leve, IAN moderada e IAM grave. Para fins de dicotomização, o escore EBIA final foi recategorizado como: SAN ou IAN (agrupados: insegurança alimentar leve, moderada ou grave).

As variáveis do estudo foram demonstradas por meio de estatística descritiva. O teste de Shapiro Wilk foi aplicado para distribuição de normalidade. Os testes de teste de qui-quadrado, T de Student e Mann Whitney, além do cálculo de regressão logística múltipla tendo como desfecho binário a presença de SAN, utilizando as diversas variáveis estudadas: categoria de fórmula infundida, faixa etária, sexo, via de acesso, vínculo do cuidador, beneficiário de algum programa governamental, entre outras foram aplicados para associar os dados. O critério definido para a inclusão das variáveis, para posterior ajuste, foi a associação com a variável dependente (SAN) na análise bivariada com um valor de "p" deveria ser inferior a 0,20 . As variáveis foram incluídas na análise de regressão pelo método Enter, de acordo com o valor decrescente da razão de prevalências (ORIC95\%). O nível de significância utilizado foi de 5\% (p<0,05). A análise estatística foi realizada por meio do programa estatístico Statistical Package for Social Sciences (SPSS Inc, Chicago, IL, EUA) versão 19.0.

\section{Resultados}

Fizeram parte da pesquisa 45 indivíduos, onde 64,4\% (n=29) do sexo feminino, além da grande maioria ser composta por idosos $(86,7 \% ; \mathrm{n}=39)$. A idade média foi de aproximadamente 75 anos e a renda familiar teve amplitude de 1 a 25 salários mínimos com renda per capita média de 1,12 salários. O tempo de diagnóstico da doença variou entre 1 e 240 meses e o tempo em TNED de 1 a 132 meses (Tabela 1). A TNED teve início aproximadamente 28 meses após o AVC.

Tabela 1. Dados socioeconômicos e clínicos dos indivíduos em terapia nutricional enteral domiciliar.

\begin{tabular}{|c|c|c|c|c|}
\hline Variáveis & Média/desvio padrão & Mediana & Mínimo & Máximo \\
\hline Média de Idade (anos) & $74,56 \pm 11,86$ & 76 & 43 & 94 \\
\hline Renda Familiar $\left(\mathrm{SM}^{*}\right)$ & $4,15 \pm 4,58$ & 3 & 1 & 25 \\
\hline Renda per capta $\left(\mathrm{SM}^{*}\right)$ & $1,12 \pm 1,06$ & 0,86 & 0,2 & 6,2 \\
\hline Número de pessoas no domicílio & $4,18 \pm 2,57$ & 4 & 2 & 14 \\
\hline Tempo de diagnóstico (meses) & - & 36 & 1 & 240 \\
\hline Tempo de TNED (meses) & - & 21 & 1 & 132 \\
\hline
\end{tabular}

Notas: *SM= salários mínimos; TNED= Terapia Nutricional Enteral Domiciliar.

Fonte: Autores.

Conforme exposto na Tabela 2, 30 indivíduos eram analfabetos ou com ensino fundamental incompleto (15,6\% e 51,1\%, respectivamente); 46,7\% eram casados e 42,2\% eram viúvos. Aproximadamente $18 \%$ eram beneficiários de programa governamental e 15,6\% recebiam pensão; 88,9\% eram aposentados e tinham como cuidador um familiar, o cônjuge (20\%) ou o(a) filho(a) $(62,2 \%)$. 
Tabela 2. Variáveis socioeconômicas dos indivíduos com acidente vascular encefálico em terapia nutricional enteral domiciliar.

\begin{tabular}{|c|c|c|c|}
\hline Variável & Níveis & $\mathbf{n}$ & $\%$ \\
\hline \multirow[t]{6}{*}{ Escolaridade } & Analfabeto & 7 & 15,6 \\
\hline & Ensino fundamento incompleto & 23 & 51,1 \\
\hline & Ensino fundamento completo & 8 & 17,8 \\
\hline & Ensino médio incompleto & 2 & 4,4 \\
\hline & Ensino médio completo & 1 & 2,2 \\
\hline & Ensino Superior & 4 & 8,9 \\
\hline \multirow[t]{4}{*}{ Etnia } & Branco & 31 & 71,1 \\
\hline & Negro & 3 & 6,7 \\
\hline & Pardo & 7 & 15,6 \\
\hline & Amarelo & 3 & 6,7 \\
\hline \multirow[t]{4}{*}{ Estado Civil } & Solteiro (a) & 2 & 4,4 \\
\hline & Casado (a) & 21 & 46,7 \\
\hline & Viúvo (a) & 16 & 42,2 \\
\hline & Divorciado (a) & 3 & 6,7 \\
\hline \multirow[t]{2}{*}{ Aposentado } & Sim & 40 & 88,9 \\
\hline & Não & 5 & 11,1 \\
\hline Beneficiário de algum programa & Sim & 8 & 17,8 \\
\hline governamental & Não & 37 & 82,2 \\
\hline \multirow[t]{4}{*}{ Cuidador } & Informal/Familiar & 37 & 82,2 \\
\hline & Esposo (a) & 9 & 20 \\
\hline & Filhos & 28 & 62,2 \\
\hline & Formal/Contratado & 8 & 17,8 \\
\hline
\end{tabular}

Fonte: Autores.

Foi notado que 46,7\% $(\mathrm{n}=21)$ dos domicílios apresentavam IAN e todos foram categorizados com IAN leve. Quando relacionados os dados, somente a idade e renda per capita foram significativamente diferentes quando comparados os perfis dos domicílios com a dicotomização de SAN (p<0,05) (Tabela 3).

Tabela 3. Comparação das variáveis socioeconômicas, clínicas, antropométricas e dietéticas com a segurança alimentar e nutricional entre indivíduos em terapia nutricional enteral domiciliar.

\begin{tabular}{lccc}
\hline & SAN (n=24) & IAN (n=21) & Valor de p \\
\hline Idade (anos) & $79,75 \pm 9,93$ & $68,62 \pm 11,25$ & $0,01^{\mathrm{a}}$ \\
Renda Familiar (SM) & $4,75 \pm 5,11$ & $3,47 \pm 3,90$ & $0,14^{\mathrm{b}}$ \\
Renda per capita (SM) & $1,38 \pm 1,27$ & $0,82 \pm 0,66$ & $0,04^{\mathrm{b}}$ \\
Tempo de diagnóstico (meses) & $68,50 \pm 58,07$ & $48,71 \pm 41,34$ & $0,23^{\mathrm{b}}$ \\
Tempo de TNED (meses) & $33,92 \pm 29,83$ & $23,50 \pm 28,91$ & $0,15^{\mathrm{b}}$ \\
\hline
\end{tabular}

Notas: IAN = Insegurança Alimentar e Nutricional; SAN= Segurança Alimentar e Nutricional; TNED=Terapia Nutricional Enteral Domiciliar.

${ }^{\mathrm{a}}$ Teste T de Student para dados paramétricos; ${ }^{\mathrm{b}}$ Teste de Mann Whitney para dados não-paramétricos.

Fonte: Autores. 
Conforme exposto no Gráfico 1, ao relacionar a SAN com a categoria da fórmula oferecida, a IAN predominou nos indivíduos que receberam fórmula mista e a SAN nos doentes que utilizaram fórmula industrializada e com alimentos.

Gráfico 1. Categoria de fórmula comparada com o nível de segurança alimentar e nutricional.

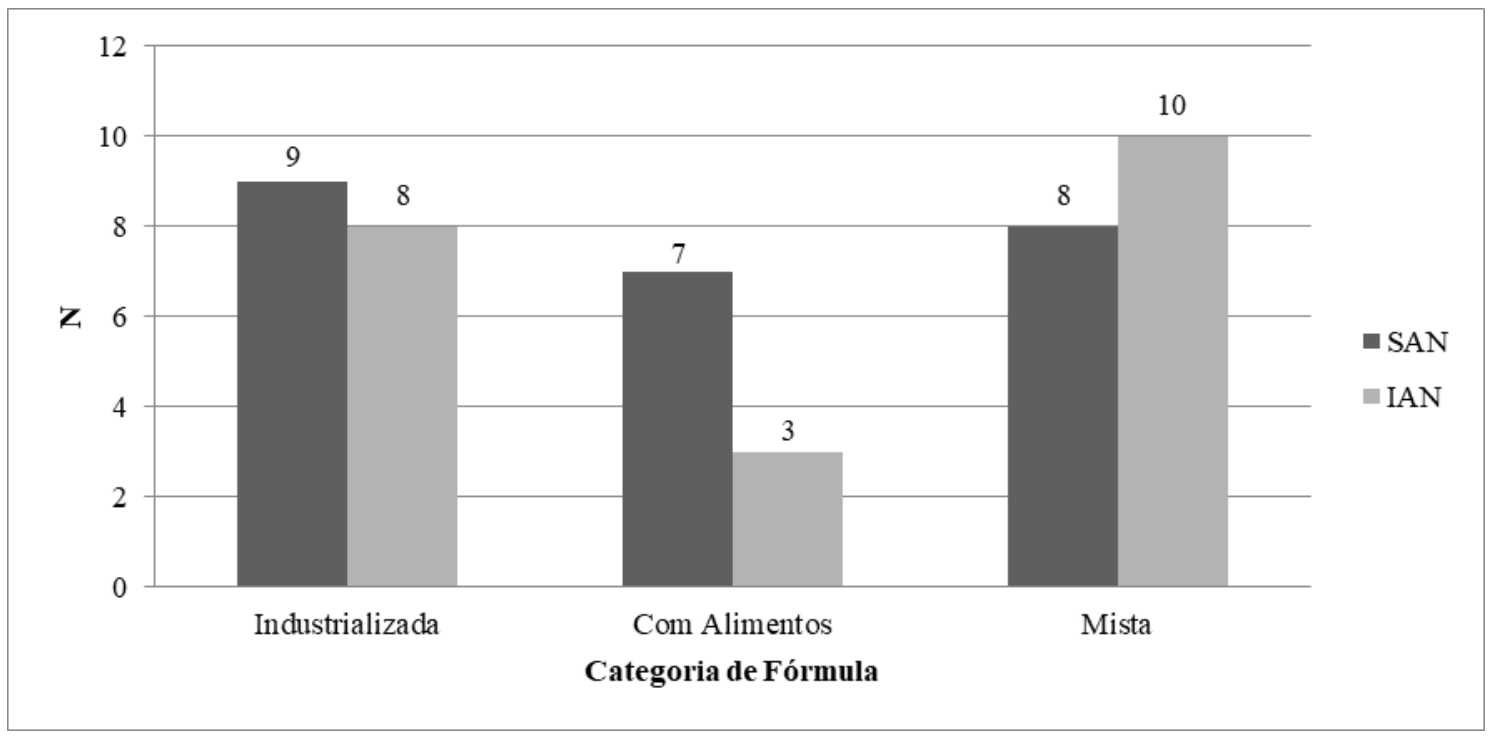

Notas: SAN= Segurança Alimentar e Nutricional; IAN= Insegurança Alimentar e Nutricional.

Fonte: Autores.

Na comparação entre SAN e as variáveis socioeconômicas foi registrado que não houve associação entre as variáveis (Tabela 4)

Tabela 4. Comparação de segurança alimentar com variáveis socioeconômicas e do estado nutricional de indivíduos em terapia nutricional enteral domiciliar.

\begin{tabular}{|c|c|c|c|c|}
\hline & $S A N(n=24)$ & $I A N(N=21)$ & OR-IC & Valor de p* \\
\hline \multicolumn{5}{|l|}{ Sexo } \\
\hline Feminino & 17 & 12 & $0,55(0,16-1,88)$ & \multirow{2}{*}{0,37} \\
\hline Masculino & 7 & 9 & - & \\
\hline \multicolumn{5}{|l|}{ Faixa etária } \\
\hline Adulto & 1 & 5 & - & \multirow{2}{*}{0,08} \\
\hline Idoso & 23 & 16 & $0,14(0,06-1,31)$ & \\
\hline \multicolumn{5}{|l|}{ Aposentadoria } \\
\hline Sim & 22 & 18 & $1,88(0,28-12,19)$ & \multirow{2}{*}{0,65} \\
\hline Não & 2 & 3 & - & \\
\hline \multicolumn{5}{|c|}{ Benificiário de programa Governamental } \\
\hline Sim & 3 & 5 & - & \multirow{2}{*}{0,44} \\
\hline Não & 21 & 16 & $0,46(0,09-2,20)$ & \\
\hline \multicolumn{5}{|c|}{ Cuidador familiar } \\
\hline Sim & 19 & 17 & $0,89(0,21-3,88)$ & \multirow{2}{*}{0,59} \\
\hline Não & 5 & 4 & - & \\
\hline
\end{tabular}




\begin{tabular}{ccccc}
\hline Renda Familiar & & & & \\
\hline \\
$\leq 2,5 \mathrm{SM}$ & 9 & 12 & $0,45(0,14-1,49)$ & 0,19 \\
$>2,5 \mathrm{SM}$ & 15 & 9 & & 0,04 \\
\hline Renda per capta & 14 & 18 & $0,23(0,05-1,02)$ & - \\
\hline 1 SM & 10 & 3 & & \\
$>1$ SM & 10 & & & \\
\hline
\end{tabular}

Nota: $\mathrm{OR}=$ Razão de chances; $\mathrm{SM}=$ salário mínimo equivalente a $\mathrm{R} \$ 678,00$ (ano base 2013).

*p é relativo ao teste de qui-quadrado.

Fonte: Autores.

Na Tabela 5 é possível notar que a SAN preexistente nos domicílios dos doentes não foi considerada variável preditora independente para a categoria de fórmula administrada de acordo com regressão logística múltipla, mesmo quando ajustado para outras variáveis $(\mathrm{p}>0,05)$.

Tabela 5. Análise de regressão a partir da dependência de segurança alimentar e nutricional

\begin{tabular}{lccc}
\hline Fator & B valor & p valor & OR (IC95\%) \\
\hline Fórmula industrializada & 0,349 & 0,729 & $1,42(0,19-10,22)$ \\
Fórmula com alimentos & 0,551 & 0,639 & $1,73(0,17-17,27)$ \\
Fórmula mista & $0^{\mathrm{b}}$ & - & - \\
Cuidador familiar & 0,67 & 0,625 & $1,96(0,13-28,90)$ \\
Benificiário de algum programa & $-1,47$ & 0,258 & $0,23(0,02-2,94)$ \\
governamental & & & $0,85(0,13-5,48)$ \\
Sexo masculino & $-0,16$ & 0,868 & $1,75(0,07-45,25)$ \\
Idosos & 0,56 & 0,736 & \\
\hline
\end{tabular}

Fonte: Autores.

\section{Discussão}

A avaliação da SAN aplicada nesta pesquisa demonstrou que a maioria dos domicílios estava em SAN, e a IAN, quando presente, foi classificada em sua totalidade com nível leve. Não houve relação entre a SAN e a categoria de fórmula administrada, nem com o sexo e a faixa etária. É importante salientar que este é o primeiro estudo que associou um instrumento determinante de SAN e TNED. A SAN não deve ser considerada como somente o resultado do instrumento de avaliação EBIA, mas também como o resultado da adequação nutricional em relação às necessidades e à premissa da garantia do Direito Humano à Alimentação e Nutrição Adequadas relativo ao acesso ao alimento/fórmula (Thieme et al., 2021). Desta maneira, recomenda-se a extrapolação da EBIA para avaliação de dados socioeconômicos e nutricionais como um todo.

Em relação ao tipo de fórmula utilizada na TNED foi visto que nas famílias em SAN prevaleceu o oferecimento da fórmula comercial; enquanto nas famílias em IAN a maioria recebeu formulação mista. Sabe-se que as fórmulas comerciais em sua maioria eram reconstituídas com água e também havia pouca variação. A categoria de fórmula mais utilizada pelos doentes no geral foi a mista. A categoria de fórmula é muito discutida entre os profissionais da saúde; o argumento é de que na fórmula comercial possui menos manipulação, composição nutricional definida e que, desta forma, ela seria segura sanitariamente (Freijer et al., 2014; Mezzomo et al., 2021). Para a escolha da fórmula alimentar deve-se levar em consideração a situação clínica do doente, as características das fórmulas, a higiene do domicílio e dos equipamentos necessários para o preparo e a condição 
socioeconômica da família, considerando que existem outros gastos associados, como: frasco, equipo, fraldas, medicamentos, etc além da condição de SAN (MAZUR et al., 2014). Desta maneira, o custo da fórmula pode pesar no orçamento, contribuindo para a opção pela formulação com alimentos e possível desfecho com a SAN (Mezzomo et al., 2021).

Entretanto, quando comparada a SAN com as diferentes categorias de fórmula também não foi observada associação. É preciso ressaltar que pode haver prejuízos nutricionais nas fórmulas com alimentos ou mistas, pois há muitas perdas na preparação.

Assim, o provimento das fórmulas nutricionais adequadas ao quadro clínico é parte do sucesso da TNED. O monitoramento da TNED é característica importante para a garantia do alcance das necessidades do paciente, oportuniza a avaliação do plano de tratamento e a deteç̧ão precoce de complicações (Elia \& Stratton, 2008; Ojo \& Brooke, 2016).

Por fim, os programas de alimentação e nutrição para doentes em TNED devem considerar a situação individual como o diagnóstico clínico, estado nutricional, situação socioeconômica e cultural, além da SAN.

\section{Conclusão}

As famílias dos indivíduos com AVC em TNED apresentam em sua maioria SAN, e a IAN, quando presente, foi classificada com nível leve. $\mathrm{O}$ uso de fórmulas preparadas com alimentos ou industrializadas não mostra diferença na classificação de SAN.

Faz-se necessário que o poder público, por meio de programas e políticas públicas, regularize a TNED incluindo-a em seu planejamento e orçamentos, garantindo o Direito Humano à Alimentação e Nutrição Adequadas para a população em situações especiais em que o risco de IAN possa ser maior. Sugere-se a condução de pesquisas nessa temática para complementar os achados desse estudo.

\section{Agradecimentos}

Agradecemos a Prefeitura Municipal de Curitiba, especialmente à Secretaria Municipal de Saúde pela concessão do campo de pesquisa.

\section{Referências}

American Dietetic Association (ADA) (2013). Position of the Academy of Nutrition and Dietetics: Functional Foods. Eat Right 113(8), 1096-1103. doi: 10.1016/j.jand.2013.06.002

Bickel, G., Nord, M., Price, C., Hamilton, W., Cook, J. (2000) Guide to measuring household food security: revised 2000. Alexandria: United States Department of Agriculture, Food and Nutrition Service.

Elia, M., Stratton, R. J. (2008). A cost-utility analysis in patients receiving enteral tube feeding at home and in nursing homes. Clinical Nutrition, $27,416-423$.

Freijer, K., Bours, M. J. L., Nuijten, M. J. C., Poley, M. J., Meijers, J. M. M., Halfens, R. J. G., Schols, J. M. G. A. (2014). The Economic Value of Enteral Medical Nutrition in the Management of Disease-Related Malnutrition: A Systematic Review. JAMDA, 15(1), 17-29. doi:10.1016/j.jamda.2013.09.005

Kepple, A. W.; Segall-Corrêa, A. M. (2011). Conceituando e medindo segurança alimentar e nutricional. Ciência \& Saúde Coletiva, Rio de Janeiro, 16(1), 187199. doi:10.1590/S1413-81232011000100022

Mazur, C. E., Schmidt, S. T., Rigon, S. A., Schieferdecker MEM (2014). Terapia nutricional enteral domiciliar: interface entre direito humano à alimentação adequada e segurança alimentar e nutricional. Demetra - Alimentação, Nutrição e Saúde, 9(3), 757-769. doi:10.12957/demetra.2014.10345

Mazur, C. E., Pinheiro, P. A. R. G., Soares, M. M. A., Thieme, R. D., Maluf, E. M. C. P., Schieferdecker MEM (2021). Nutritional composition of the enteral formulas used in home enteral nutrition. International Journal of Development Research, 11(18), 50910-50913. doi:10.37118/ijdr.23138.10.2021

Mezzomo, T. R., Fiori, L. S., Reis, L. O., Schieferdecker, MEM (2021). Nutritional composition and cost of home-prepared enteral tube feeding. Clinical Nutrition Espen, 42, 393-399. doi:10.1016/j.clnesp.2020.12.016

Mizzuno, P. B., Costa, S. M., Arana, A. R. A., Uliana, M. R. (2021). Políticas de segurança alimentar e nutricional no Brasil e sua relação com a agricultura familiar. Campo-Território: Revista de Geografia Agrária, 16(40), 91-114. doi:10.14393/RCT164004

Ojo, O., Brooke, J. (2016). The Use of Enteral Nutrition in the Management of Stroke. Nutrients, 8(12), 827. doi: 10.3390/nu8120827 
Research, Society and Development, v. 10, n. 15, e141101522879, 2021

(CC BY 4.0) | ISSN 2525-3409 | DOI: http://dx.doi.org/10.33448/rsd-v10i15.22879

Perez-Escamilla, R., Segall-Corrêa, A. M., Maranha, L. K., Sampaio, M. F. A., Marín-León, L., Panigassi, G. (2004). An adapted version of the U.S. Department of Agriculture Food Insecurity Module is a valid tool for assessing household food insecurity in Campinas, Brazil. Journal of Nutrition, 135, 1923-1928. doi: $10.1093 / \mathrm{jn} / 134.8 .1923$

Poulain, J. P. (2004). Sociologias da Alimentação. Florianópolis: Editora da UFSC.

Thieme, R. D., Schieferdecker, M. E. M., Ditterich, R. G. (2021) Políticas públicas e o direito à saúde e à alimentação de crianças e idosos em terapia nutricional enteral em domicílio. Revista de Direitos Sociais e Políticas Públicas, 9(2), 689-730. doi:10.25245/rdspp.v9i2.971

Vasconcellos, A. B. P.; Moura, L. B. A. (2018). Segurança alimentar e nutricional: uma análise da situação da descentralização de sua política pública nacional. Cadernos de Saúde Pública, 34(2), 1-13. doi:10.1590/0102-311X00206816 